\title{
Model of Laser-Induced Nano-Cavitation in the Surface of Gelatin Confronted with the Fast Rise Time of the Optical Attenuation Measurements
}

\author{
Sylvain Lazare ${ }^{1}$ \\ ${ }^{1}$ Institut des Sciences Moléculaires (ISM) UMR 5255, Université Bordeaux 1, Talence, France \\ Correspondence: Sylvain Lazare, Institut des Sciences Moléculaires (ISM) UMR 5255, Université Bordeaux 1, \\ 351 cours de la Libération, Talence 33405, France. Tel: 33-540-006-975. E-mail: s.lazare@ism.u-bordeaux1.fr
}

Received: February 16, 2013 Accepted: March 19, 2013 Online Published: March 31, 2013

doi:10.5539/apr.v5n3p1 URL: http://dx.doi.org/10.5539/apr.v5n3p1

\begin{abstract}
In the laser-polymer interaction a particular mechanism is evidenced when the polymer absorption coefficient is small. In this case the laser ablation is most of the time, but not necessarily, accompanied by intense bubbling, which results from the interplay between ablation gas, polymer melt and pressure wave. In this work, the inception of this phenomenon called cavitation, is monitored for the gelatin case (as a model polymer) by the fast extinction of a probe cwHeNe laser for several fluences of the $\operatorname{KrF}$ laser pulses $\left(1.13,0.65,0.470 \mathrm{~J} / \mathrm{cm}^{2}\right)$ used to trigger ablation whose threshold is $0.5 \mathrm{~J} / \mathrm{cm}^{2}$. A new model combining the time dependence of temperature, pressure and viscosity is developed for the simulation of this extinction due to the cavitation phenomenon and used to fit the experimental data. The model concludes that the cavity precursors with initial radius $\mathrm{R}_{0}=3 \mathrm{~nm}$ and concentration $\mathrm{n}_{0}=5 \times 10^{13} \mathrm{~cm}^{-3}$ are suddenly expanded by the tension wave launched immediately after the absorption of the laser pulse. The probe laser radiation is attenuated by Rayleigh or Mie scattering with a rise time of the order of $110 \mathrm{~ns}$, depending on the ablation fluence, and corresponding to the transit of the tension wave. The dynamics of the radius increase is given by the Rayleigh-Plesset equation and the thermodynamic kinetics of the growth is given by the classical theory of the homogeneous nucleation. The model shows that complete darkening of the sample surface is achieved only for a fluence larger than $1.1 \mathrm{~J} / \mathrm{cm}^{2}$ and that the increase of the radius is limited to the range 3-100 nm, in this case. The approximations are discussed in the paper. In the experiments, the increase of the opacity of the sample surface occurs also at lower fluence ( 0.65 and $0.470 \mathrm{~J} / \mathrm{cm}^{2}$ ) although with a slower dynamics. This reveals that another mechanism may be invoked to account for the slower opacity rise observed at lower fluences in the experimental data. The model excludes melting in these cases and indicates rather a solid phase transition due to the gas evolution.
\end{abstract}

Keywords: laser, gelatin, surface, foaming, cavitation, model, attenuation, transient

$\begin{array}{ll}\text { Nomenclature } & \\ A & \text { surface absorptivity } \\ \alpha & \text { absorption coefficient } \\ F & \text { laser fluence density on the polymer } \\ c_{p} & \text { heat capacity } \\ \Delta T & \text { temperature elevation } \\ T & \text { temperature } \\ \lambda & \text { laser wavelength } \\ \tau & \text { main laser pulse with parameter } \\ \tau 1 & \text { second pulse width parameter } \\ m_{x} & \text { laser pulse components mixing coefficient } \\ \Delta t & \text { laser pulse second component time shift }\end{array}$


$t \quad$ time

$g(t) \quad$ laser pulse time profile

$F_{0} \quad$ incident laser fluence

$z \quad$ depth in the polymer

$\Delta T_{\max } \quad$ maximum temperature elevation

$f_{0} \quad$ instantaneous laser fluence

$c_{S} \quad$ sound velocity

$\Gamma \quad$ Grüneisen coefficient

$\Delta p \quad$ elementary pressure elevation

$\beta \quad$ coefficient of thermal expansion

$U \quad$ internal energy

V volume

$p_{1} \quad$ pressure of subwave 1

$p_{2} \quad$ pressure of subwave 2

$R_{S} \quad$ sound reflection coefficient

$g \quad$ gas fraction

$P_{\text {pol }} \quad$ pressure in the polymer

$p_{\text {pol }} \quad$ elementary pressure in the polymer

$P_{\text {gas }} \quad$ pressure of the gas system

$p_{\text {gas }} \quad$ elementary pressure in the gas system

$\Delta P \quad$ difference of pressures inside and outside the cavity

$J(z, t) \quad$ rate of nucleation

$\sigma_{0}$

$\sigma(z, t)$

surface tension of the polymer at room temperature

$T_{c}$

surface tension of the polymer

$n_{0}$

critical temperature of the polymer

$Z(z, t) \quad$ Zeldovich factor, frequency factor of the rate law

$R(z, t) \quad$ radius of the growing cavity

$R_{0} \quad$ initial radius of the growing cavity

$n_{f v h} \quad$ density of free volume holes

$v_{f v h} \quad$ volume of a free volume hole

$R_{f v h} \quad$ radius of free volume holes

$S \quad$ reducing factor for density

$v_{\text {ass }} \quad$ volume of polymer associated to a cavity

$E_{x p}(z, t) \quad$ expansion ratio

$\eta(z, t) \quad$ viscosity of the polymer

$\eta_{\infty} \quad$ viscosity at infinite temperature

$T_{R} \quad$ reference temperature in the viscosity model

$P_{R} \quad$ reference pressure the viscosity model

$\delta_{T} \quad$ temperature exponent in the viscosity model

$\delta_{p} \quad$ pressure exponent in the viscosity model

$T_{g m} \quad$ glass transition temperature of the mixture

$T_{g p} \quad$ glass transition temperature of the polymer 


$\begin{array}{ll}T_{g w} & \text { glass transition temperature of water } \\ k_{w} & \text { Gordon-Taylor parameter } \\ X_{w} & \text { water fraction } \\ X_{p} & \text { polymer fraction } \\ n_{c}(z, t) & \text { density of critical cavities } \\ \lambda_{0} & \text { probe laser wavelength } \\ n_{s} & \text { refractive index of the cavities at the probe laser wavelength } \\ n_{m} & \text { refractive index of the polymer at the probe laser wavelength } \\ m=n_{s} / n_{m} & \text { refractive index ratio } \\ A t t(t) & \text { probe laser attenuation } \\ I_{0} & \text { incident probe laser intensity } \\ I(t) & \text { probe laser intensity } \\ s(t) & \text { coefficient of attenuation by scattering } \\ E_{c}(z, t) & \text { energy of cavitation } \\ E_{i n}(z, t) & \text { input energy }\end{array}$

\section{Introduction}

The science of laser ablation won its development and popularity mainly due to its unique applicability in various fields like materials etching or synthesis, micro/nano-systems production, biomedical microprocessing, etc.. The pioneering report of Trockel et al. (1983) on Excimer Laser Surgery of Cornea was one of the most critical for its success in the field of biomaterials and tissue. More recently new femtosecond laser sources have largely amplified its success (for recent review see Plamann, 2010). Since then, polymers and biopolymers are frequently studied as prototypes (in Dorronsoro et al., 2008) for potential application in biological tissue processing (Vogel \& Venugopalan, 2003). At first, the curiosity of scientists was attracted by the so-called cleanliness of ultraviolet (UV) laser ablation (LA), a clean cut or etching, well defined by the small size of the beam spot on the surface of the material. Such approach was even proposed for subcellular nanosurgery by using the radiation femtosecond laser in Vogel (2005). In the experiments, this highly efficient phenomenon is achieved e.g. in the ArF laser ablation of cornea $(\lambda=193 \mathrm{~nm})$ (Pettit \&Ediger 1996, Yablon et al., 1999, Fisher \& Hahn, 2004), when the polymer absorption coefficient is high $\alpha \approx 10^{4}$ to $10^{5} \mathrm{~cm}^{-1}$ (depending on the water content of the target) and when the incident fluence is significantly above the ablation threshold $F \gg F_{T}$. In this case the energy density input in the surface evaluated as $A \alpha F=\rho c_{p} \Delta T$ is very high, corresponding to a temperature elevation of $\Delta T=\frac{1 * 10^{5} * 2}{1 * 2} \approx 10^{4}-10^{5} \mathrm{~K}$ (standards values in usual units are typically $\rho=1$ for the density, $c_{p}=2$ for the specific heat capacity, $\mathrm{A}=1$ for the absorptivity and $F=2$ for the incident fluence). The equivalent average available thermal energy in molecular scaling is then of the order of $k T=8.5 \mathrm{eV}(k$ Boltzmann constant), that is to say by far in excess when compared to the covalent bond energy of the polymer ( 3 to $5 \mathrm{eV}$ ). Clean ablation is then due to the fast bond breaking and gas formation in most of the absorbing volume and is therefore easily predictable in this scenario. It is more complex for opposite situations where the absorption coefficients are limited to small values in the range of 1 to $10^{3} \mathrm{~cm}^{-1}$ (e.g. biopolymers at $\lambda=248 \mathrm{~nm}$ ). The same molecular thermal energy is not above $k T=0.1 \mathrm{eV}$, a value which is far below the onset of thermal bond breaking in the same condition of fluence. In this case, a new type of ablative phenomena, often called "mechanical ablation" (Paltauf, 2003) or "cold ablation" (Oraevsky et al., 1996) because the temperature elevation is small $\left(\Delta T \leq \frac{0.1 \mathrm{eV}}{k}=1000 \mathrm{~K}\right)$ compared with the "high temperature mechanism" $\left(\Delta T \approx \frac{8.5 \mathrm{eV}}{k}=10^{5} \mathrm{~K}\right)$, can be observed at low fluence thanks to a combination of various mechanisms among which the role of the strong laser-induced pressure-and-tension wave is critical. In this work such new type of mechanism is studied with dry gelatin films $\left(\alpha=600 \mathrm{~cm}^{-1}\right.$ at $\left.\lambda=248 \mathrm{~nm}\right)$ as a convenient model polymer. Gelatin is industrially extracted from various animal tissues rich in collagen by mild hydrolysis (for a recent review see Gómez-Guillén et al., 2011) and easily available in the form of dry film $(\approx 200 \mu \mathrm{m}$ thick with $4 \%$ of water $)$ with smooth surfaces suitable for optical measurements. Its primary chemical structure is therefore very similar to that of collagen, the main constituent of the cornea tissue. It is mostly composed of protein chains having the capability to form gels with water, in particular owing to the winding capability of three chain end into small triple helix and forming easily three dimensional networks. In dry films the polymer molecules are mainly randomly organized and their 
thermal properties are assumed to be similar to those of an amorphous polymer (Roussenova et al., 2012). Laser-induced foamy layers on polymer and biopolymer surfaces have been published first in Lazare et al. (2005) and since then reported by other groups (Castillejo et al., 2012). The dynamics of the foaming phenomenon is measured experimentally by monitoring, in a time-window of 0 to $1 \mu \mathrm{s}$ after the laser pulse (width $\approx 40 \mathrm{~ns}$ ), the rise of the attenuation of a HeNe laser beam transmitted by a gelatin film (Lazare et al., 2009). The rise of the attenuation occuring after the absorption of the ablation laser pulse is attributed to the scattering of the light by the growing nano-cavities formed at the inception of bubbling. By this method the mechanisms of the laser-induced bubbling can be studied and modeled. The experimental rise curves in Figure 1 are then compared with simulated curves obtained with the aid of the recent model of the tension wave (Lazare et al., 2009, 2010) in the melted layer of polymer formed at the surface of the gelatin film. Our kinetic model indicates that the tension-assisted cavitation takes place when the $\mathrm{KrF}$ laser fluence is larger than $1.1 \mathrm{~J} / \mathrm{cm}^{2}$ and predicts a short rise time in fair agreement with the fast experimental one of $\approx 110 \mathrm{~ns}$. However for the two fluences below 1.1 $\mathrm{J} / \mathrm{cm}^{2}$, the predicted cavitation growth and the corresponding rise of attenuation are negligible. Therefore another mechanisms of cavity growth is then necessary to account for the slower attenuation rise times of $\approx 900 \mathrm{~ns}$ and $\approx$ $3500 \mathrm{~ns}$ measured in the experiments.

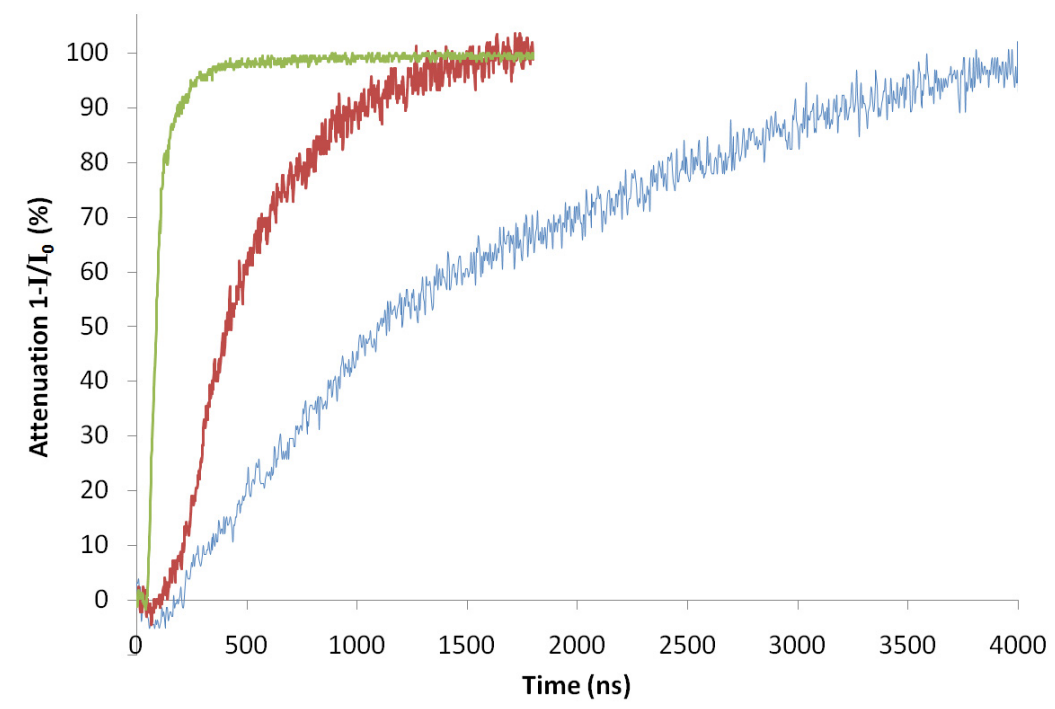

Figure 1. Probe HeNe beam attenuation rise curves for the 3 fluences used: from the fastest to the slower: 1.130 $\mathrm{J} / \mathrm{cm}^{2}, 0.650 \mathrm{~J} / \mathrm{cm}^{2}$ and $0.470 \mathrm{~J} / \mathrm{cm}^{2}$. The attenuation is due to the light scattering by the growing nano-cavities, precursors of bubbles under the action of the tension wave

\section{Materials and Methods}

The studied gelatin films were purchased from Merck and used without any particular preparation. Their water content is related to the atmospheric humidity and is estimated in the range of 5-8\%. The experimental setup developed to measure the fast attenuation rise curves is described in Lazare et al. (2009). The computer programs were written using either MathCad (Math Soft) or the SCILAB language freely available from the site of Scilab Enterprises (Versailles, France).

\section{Pressure Wave Model}

The pressure wave model has been presented in several papers (Lazare et al., 2009, 2010; Lazare, 2010) and a new improved version is presented in details here using essentially the same nomenclature (see below). The main new feature is the consideration of the pressure of the ablation gas which is important in the growth dynamics of the cavities since gas diffuse rapidly in the inner of the cavities and contribute to their expansion and stability. We consider a simulated incident laser pulse of fluence given by Equation (1) where $t$ is the time. It approaches the experimental time profile of the pulse of the KrFexcimer laser (Lambda Physik 220i) (see Lazare et al., 2009) by adjusting the various parameters $\tau=7.5 \mathrm{~ns}, \tau_{1}=9 \mathrm{~ns}, m_{x}=0.15$ and $\Delta t=45 \mathrm{~ns}$. It is composed of two main peaks of intensity shifted by a time interval of $\Delta t=45 \mathrm{~ns}$, represented by the normalized sum of two functions mixed with the coefficient $m_{x}=0.15$. The second gaussian peak is important to be considered since it plays a significant role on the intensity of the tension wave (Figure 3) and indirectly on the swiftness of the 
cavitation phenomenon.

$$
F(t)=F_{0} \times\left[\int_{0}^{\infty} g\left(t^{\prime}\right) d t^{\prime}\right]^{-1} \times \int_{0}^{t} g\left(t^{\prime}\right) d t^{\prime}, g(t)=m_{x} \times t^{2} e^{-\frac{t}{\tau}}+\left(1-m_{x}\right) \times e^{-\frac{\ln (2)(t-\Delta t)^{2}}{\tau 1^{2}}}
$$

By absorption of the incident pulse of light, the temperature profile as a function of time is given by Equation (2) in which $z$ is the depth into the surface. $A, c_{p}$ and $\rho$ are respectively the absorptivity ( $A=0.94$ for gelatin), the heat capacity and the density of the target polymer.

$$
T(z, t)=\frac{\alpha A}{c_{p} \rho} F_{0} e^{-\alpha z}+T_{0}=\Delta T_{\max } e^{-\alpha z}+T_{0}
$$

In these experiments the temperature profile is considered as invariant in the pertinent time window starting at the end of the laser pulse up to $\approx 4 \mu$ s due to the slow heat diffusion in the polymer. The subsequent pressure elevation is considered since in the timescale of nanoseconds the polymer cannot relax fast enough. We consider first the short elementary pulse case of fluence $f_{0}$ and then the real long pulse case is obtained later by adding the effects of many short pulses simulating the real long laser pulses. In the following, by convention lower case letters $f, p$ mean elementary quantities whereas upper case $F$ and $P$ mean integrated quantities. Pressure relaxation takes place mainly by a wave-type propagation, usually well described by equation of propagation (3), where $c_{s}$ is the celerity of sound (pressure wave or oscillation), $c_{p}$ is the heat capacity and $\beta$ is the thermal expansion coefficient of the polymer. For gelatin a realistic numerical value of $c_{s}$ is $1-1.5 \mu \mathrm{m} / \mathrm{ns}$ and $c_{p}=1.5$ $\mathrm{J} / \mathrm{g} . \mathrm{K}$ is assumed constant over the range of $T$ and $P$.

$$
\frac{\partial^{2} P(z, t)}{\partial^{2} t}-c_{S}^{2} \nabla^{2} P(z, t)=-\frac{\beta}{C_{P}} \frac{\partial[A \alpha F(z, t)]}{\partial t}
$$

From the temperature profile, the pressure elevation during the laser pulse absorption, is deducted with the aid of the Grüneisen equation of state (4), using $\Gamma$ a constant of proportionality (Equation 5) between the two related increments $\Delta p$ and $\Delta T$. After the end of the elemetary pulse $\delta \tau$ only relaxation occurs.

$$
\begin{gathered}
\Delta p(z, t=\delta \tau)=\Gamma \cdot \Delta T=\Gamma \cdot \alpha \cdot f_{0} \cdot e^{-\alpha \cdot z}=p_{\max } \cdot e^{-\alpha \cdot z} \\
\Gamma=-\left(\frac{\partial \ln P}{\partial \ln (U / V)}\right)_{V}=\frac{c_{s}^{2} \beta}{c_{p}}
\end{gathered}
$$

In practice and by approximation $\Gamma$ is taken equal to 1 for reasons which can be found in our previous papers and in (Albagli, 1994). In the present model having only one dimension, the pressure relaxation takes place in the form of two waves $p_{1}(z, t)$ and $p_{2}(z, t)$ counter-propagating along a direction perpendicular to the surface which is also that of the laser beam (Figure 2). Then two systems can be distinguished, the polymeric solid and the gas system since they do not behave completely similarly. The gas system does not stand any tension at the considered temperature, therefore wave reflection on the solid-air interface is impossible. On the contrary as long as the temperature allows it, the polymer molecules can be elongated elastically in a negative pressure and reflection then occurs when the wave transits at the solid surface. As a consequence the total pressure wave must be decomposed into the several wavelets (see Figure 2). For the polymer we consider the Equations (6) to (8) with $g$ being the fraction of ablation gas. Equation (8) is due to the wave reflection at the polymer interface with $\mathrm{Rs}=-1$ as reflection coefficient.

$$
\begin{gathered}
p_{1 p}(z, t)=0.5(1-g) \times p_{\max } e^{-\alpha\left(z-c_{s} t\right)}, \text { if } z>c_{s} t, \text { otherwise } 0 \\
p_{2 p}(z, t)=0.5(1-g) \times p_{\max } e^{-\alpha\left(z+c_{s} t\right)}, \text { if } z>0, \text { otherwise } 0 \\
p_{2 R p}(z, t)=0.5(1-g) \times p_{\max } R_{s} e^{-\alpha\left(c_{s} t-z\right)}, \text { if } c_{s} t>z>0, \text { otherwise } 0
\end{gathered}
$$

For the gas system, the two Equations (9) and (10) are similar to (6) and (7) but the third one disappears due to the absence of reflection at the solid-air surface. Typical gas fractions in the range of $g=0.1-0.5$ are considered meaning approximately that at the end of the laser pulse the ablation gases contribute to an extend $g$ to the total pressure. The wave propagation celerity $c_{\mathrm{s}}$ is the same for both systems.

$$
\begin{gathered}
p_{1 g}(z, t)=0.5 g \times p_{\max } e^{-\alpha\left(z-c_{s} t\right)}, \text { if } z>c_{s} t, \text { otherwise } 0 \\
p_{2 g}(z, t)=0.5 g \times p_{\max } e^{-\alpha\left(z+c_{s} t\right)}, \text { if } z>0, \text { otherwise } 0
\end{gathered}
$$

The resultant pressures due to a single elementary laser pulse of fluence $f_{0}$ in the polymer (see Figure 2 ) and in 
the gas system can be expressed as sum of the corresponding subwaves respectively as in Equation (11) and (12).

$$
\begin{gathered}
p_{\text {pol }}(z, t)=p_{1 p}(z, t)+p_{2 p}(z, t)+p_{2 R p}(z, t) \\
p_{\text {gas }}(z, t)=+p_{1 g}(z, t)+p_{2 g}(z, t)
\end{gathered}
$$

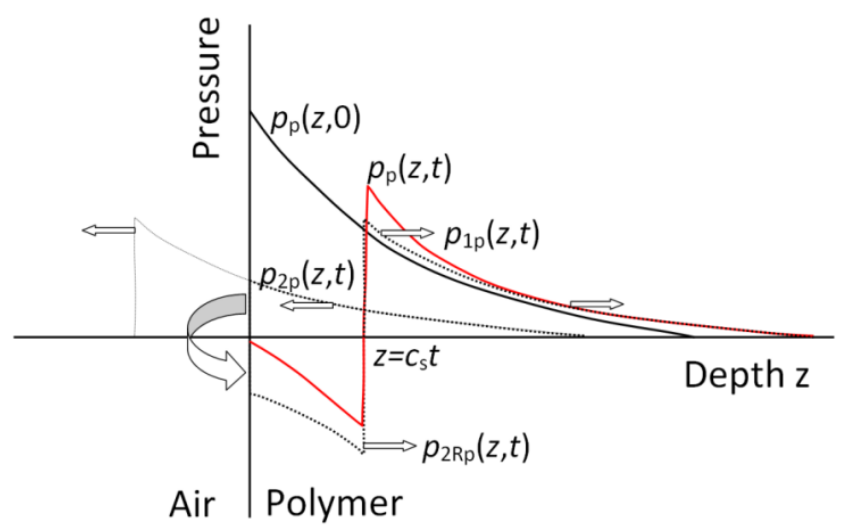

Figure 2. An elementary pressure wave $p_{\mathrm{p}}(\mathrm{z}, \mathrm{t})$ is constructed (red curve) as the sum of the two counter-propagating subwaves $p_{1 \mathrm{p}}(\mathrm{z}, \mathrm{t})$ and $p_{2 \mathrm{p}}(\mathrm{z}, \mathrm{t})$ plus the inward propagating reflected part of $p_{2 \mathrm{Rp}}(\mathrm{z}, \mathrm{t})$ of $p_{2 \mathrm{p}}(\mathrm{z}, \mathrm{t})$

The total pressure waveforms are then obtained by convoluting the elementary pressures with the pulse profile $g(t)$ as in Equations (13) and (14).

$$
\begin{gathered}
P_{\text {pol }}(z, t)=p_{\text {pol }}(z, t) \otimes g(z, t)=\int_{0}^{t} g\left(t_{1}\right) p_{\text {pol }}\left(t-t_{1}\right) d t_{1} \\
P_{g a s}(z, t)=p_{\text {gas }}(z, t) \otimes g(z, t)=\int_{0}^{t} g\left(t_{1}\right) p_{\text {gas }}\left(t-t_{1}\right) d t_{1}
\end{gathered}
$$

The driving quantity for the fast cavity growth, a non-equilibrium phenomenon, is the difference between the inner pressure of the bubbles and the outer pressure as in Equation (15).

$$
\Delta P(z, t)=P_{\text {in }}(z, t)-P_{\text {out }}(z, t)
$$

$P_{\text {in }}(z, t)$ can be easily assumed to be equal to $P_{\text {gas }}(z, t)$ since gas diffusion to the inside of the considered cavities is fast since the diffusion length is of the order of less than $10 \mathrm{~nm}$, as shown below. The value of $P_{\text {out }}(z, t)$ can be either $P_{\text {pol }}(z, t)$ if there is complete separation of polymer and gas systems (Equation 16), or more likely $P_{\text {pol }}(z, t)+P_{\text {gas }}(z, t)$ if some gas is still dissolved in the gelatin polymer (Equation 17).

$$
\begin{gathered}
\Delta_{1} P(z, t)=P_{\text {gas }}(z, t)-P_{\text {pol }}(z, t) \\
\Delta_{2} P(z, t)=P_{\text {gas }}(z, t)-\left[P_{\text {pol }}(z, t)+P_{\text {gas }}(z, t)\right]=-P_{\text {pol }}(z, t)
\end{gathered}
$$

In previous papers (Lazare et al., 2009, 2010; Lazare, 2010) we have shown that if gas pressure is not considered, the model can account for the experiment, since only the rarefaction wave and the following tension subwave are the important contributions to the cavity nucleation phenomenon. It further shows that increasing the gas fraction $g$ induces a decrease of the amplitude of the tension wave with important consequences on the rate of nucleation. $\Delta_{2} P(z, t)$ is therefore routinely used. It seems that the laser induced foaming phenomenon is mainly due to the ability of the polymer molecules to stand or generate tension (negative pressure) and to yield gas upon pulse ultraviolet excitation of photochemical reactions. It should be noted that, if negligible at the time of cavitation, gas pressure has a necessary role at long time when the polymer pressure tends to atmospheric pressure, since it stabilizes the cavity and prevents it from collapsing. 


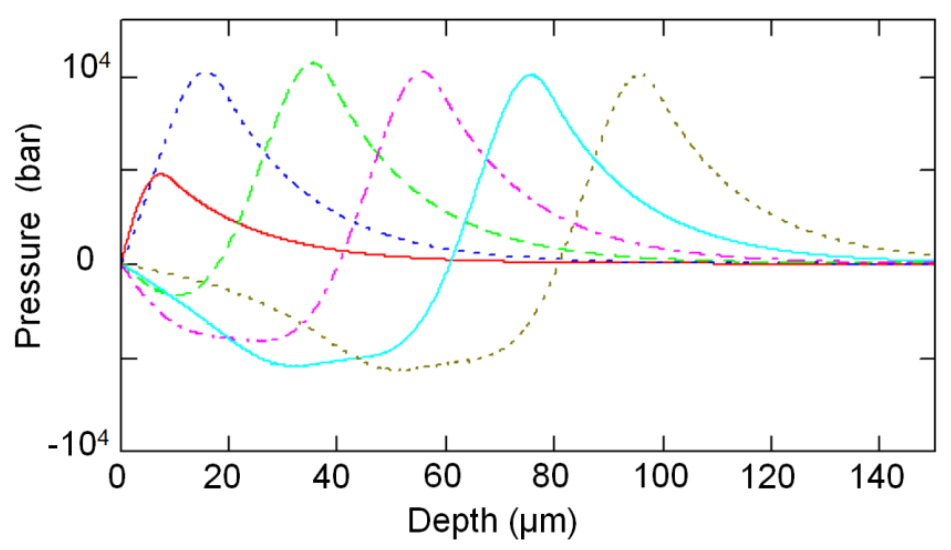

Figure 3. Pressure wave form in the polymer $P_{p o l}(z, t)$ for an incident fluence $F_{0}=1.130 \mathrm{~J} / \mathrm{cm}^{3}$, calculated with Equation (13) at various increasing times (10, 20, 40, 60, 80 and $100 \mathrm{~ns})$; other numerical values used for gelatin are given within the text and $\rho=1.20 \mathrm{~g} / \mathrm{cm}^{3}$

In the Figure 3 it is seen that the polymer tension, the important quantity for the cavity growth, starts appearing rather at the end of the laser pulse which can be roughly situated at a time larger than $35 \mathrm{~ns}$. By further propagation into the bulk its amplitude is decreased down to a minimum at time $t \approx 70 \mathrm{~ns}$ and at a depth of 30 $\mu \mathrm{m}$. However, as a rule (see below) the tension-induced cavitation takes place within the melt depth which is $13.5 \mu \mathrm{m}$ at $1.13 \mathrm{~J} / \mathrm{cm}^{2}$.

\section{Rate of the Homogeneous Nucleation and Dynamics of Cavity Radius}

The tension wave induced growth of the cavities is governed by two main equations. On one hand the dynamic model of Rayleigh-Plesset (RP) gives the increase of radius $R$ with time as a function of $P$ and $T$ and on the other hand the classical theory of the homogeneous nucleation (CTHN), the fluctuational aspect of the growth, provides the kinetics of the formation of cavities with critical radius. At first we define the term "pores" as the precursors of the cavities in the polymer before any laser excitation. The rate of critical cavities formation $J(z, t)=d n_{c} / d t$ is given by Equation (18), where $\mathrm{Z}$ is the conventional Zeldovich factor, $n_{0}$ is the initial concentration of pores (see below), $n_{c}$ is the concentration of critical cavities and $\sigma(z, t)$ is the surface tension of gelatin (19) whose temperature dependence is obtained from the Guggenheim formula (Equation 19) (for gelatin $\sigma_{0}=0.030 \mathrm{~N} / \mathrm{m}$ and $T_{c}=1000^{\circ} \mathrm{C}$ ).

$$
\begin{gathered}
J(z, t)=\frac{d n_{c}}{d t}=Z(z, t) \times\left[n_{0}-n_{c}(z, t)\right] \times \exp \left(-\frac{16 \pi \sigma(z, t)^{3}}{3 k T(z, t) \Delta P(z, t)^{2}}\right) \\
\sigma(z, t)=\sigma_{0}\left(1-T(z, t) / T_{c}\right)
\end{gathered}
$$

The Zeldovich or frequency factor $Z$ can be explicited by using Equation (20) taken from Baidakov (2007), which is well adapted to systems highly dependent on the viscosity $\eta(z, t)$. The polymer, like any solid is highly viscous at room temperature but its heating by the laser pulse and the tensilization by the pressure wave drastically decrease the viscosity in the melt depth as presented below. The cavity growth starts as soon as this decrease allows it. Therefore the phenomenon is controlled by viscosity which is an important parameter to model.

$$
Z(z, t)=\frac{\sigma(z, t)}{\eta(z, t)} \sqrt{\frac{\sigma(z, t)}{k T}}
$$

The above kinetic approach accounts for the thermal energy fluctuations allowing the critical size to be reached. Besides, when the dynamics of the cavity radius $R(z, t)$ is needed, the Rayleigh-Plesset Equation (21) (Plesset, 1949 ) yields it as a function of the medium parameters $\eta(z, t), \sigma(z, t)$ and the state parameter $P(z, t)$.

$$
R \ddot{R}+\frac{3}{2} \dot{R}^{2}+\frac{4 \eta}{\rho} \frac{\dot{R}}{R}+\frac{2 \sigma}{\rho R}=-\frac{\Delta P}{\rho}
$$

In systems where the dynamics is dominated by the viscosity parameter, this equation can be replaced by a 
simpler form (22) including only the term of viscosity and without the inertial terms. A simple solution is then given by Equation (23).

$$
\begin{gathered}
\frac{4 \eta}{\rho} \frac{\dot{R}}{R}=-\frac{\Delta P}{\rho} \\
R=R_{0} \exp \left(\frac{\Delta P}{4 \eta} t\right)
\end{gathered}
$$

This viscosity controlled exponential growth has been often used in material fracture and spallation models which were validated with the experiments (Seaman et al., 1976; Dekel et al., 1998; Kuksin et al., 2010). It is worth noting that similar approach is also used in modeling the explosive bubbling of hot lavas in volcanology (Navon et al., 1998). One major difficulty of this simulation is the choice of $R_{0}$ the initial cavity radius in Equation (23). Similarly the initial concentration $n_{0}$ of bubble precursors is equally an important and difficult choice. As explained below the simulation is guided by two important conditions to fulfill $i$ ) the close fit of the experimental attenuation rise curve given in Figure $1\left(F_{0}=1.13 \mathrm{~J} / \mathrm{cm}^{2}\right)$ and 7 and ii) the computed cavitation energy which must remain smaller than the laser input energy (see Section 7).

In our previous paper, it was suggested that the free volume holes (fvh), could play the role of these initial cavity precursors. In the case of gelatin, as reported by Akyama et al. (2007), they are present in a narrow normal distribution of radius around a mean an average value of $R_{f v h}=0.227 \mathrm{~nm}$ for dry film with a $5-8 \%$ of water content. Orthopositronium (o-Ps) $\left(\mathrm{e}^{+} \mathrm{e}^{-}\right)$annihilations spectroscopy PALS (Yampolskii, 2007) is the widely used technique to study fvh in condense matter and in polymer films in particular since their presence and properties strongly correlate with important properties like viscosity and gas permeation. Afvh is an intermolecular space in the polymer which is devoid of covalent chemical bonds and it is therefore more expandable than the surrounding polymer when the pressure becomes negative. Its expansion is achieved with much less energy comparatively, than that of a similar volume in the dense polymer containing many more bonds. Also a gas molecule can easily diffuse from solvated position into afvh overcoming a low energy barrier. In membrane science, ultrapermeable polymers with high fvh content are search for. From the point of view of fvh content gelatin is only a regular polymer with a normal content. In PALS (Yampolskii, 2010) the orthopositromium signal intensity $I_{3}$ is related to the concentration of fvh in the polymer and the o-PS lifetime $\tau_{3}$ is a measurement of $R_{f v h}$ (Tao-Eldrup relation). The method is used to study the influence of the polymer chemical structure on the fvh content. It indicates that fvh exist in a population having some radius dispersion, for simplicity we consider that every fvh have the same volume $v_{f v h}=4 / 3 \pi R_{f v h}{ }^{3}$. Similarly for the fvh concentration in gelatin we use a generic value $n_{f v h}=0.5 \times 10^{21} \mathrm{~cm}^{3}$. A justification for it is for instance provided by Table 1 in Alentiev and Yampolskii (2002) which indicates variations of fvh concentrations in a range of $n_{f v h}=$ $0.07-0.7 \times 10^{21} \mathrm{~cm}^{3}$ for a set of 9 polymers. However using this value as $n_{0}$ in the kinetic Equation (18) is no longer validated by this work (see below) because the laser-excited gelatin surface is no longer a pure polymer but rather a mixture of polymer containing the ablation gas. Then the mechanism of cavity growth is complicated by new efficient phenomena like cavity coalescence. The coalescence of several cavities into a single one is a fast and necessary mechanism which is facilitated by a lowering of the viscosity and the presence of a significant amount of ablation gas, whose diffusion is also strongly activated by the laser-induced phenomena (temperature, tension, viscosity drop). This is accounted in our model by the appearance of the effective values of initial cavity radius and concentration $\left(R_{0}, n_{0}\right)$ which are a couple of solutions which fulfill conditions $i$ ) and $\left.i i\right)$, respectively a close fit of the attenuation rise curve of Figure $1\left(F_{0}=1.13 \mathrm{~J} / \mathrm{cm}^{2}\right)$ or 7 and respect by the computed cavitation energy of the limitation imposed by the laser input energy (Figure 9). A detailed modeling of the coalescence, although desirable for a deeper understanding, is behind the scope of the present paper. Nevertheless, the free volume features are used for comparison and it is thought that there is some correlation (and maybe proportionality) between $n_{0}$ and $n_{f v h}$ as in Equation (24) in which $S$ is a reducing statistical factor accounting for the effective radius and concentration of initial expandable cavities.

$$
n_{0}=S \times n_{f v h}
$$

Another pertinent quantity in our model is the polymer volume associated to each critical cavity defined as in Equation (25).

$$
v_{\text {ass }}(z, t)=n_{c}(z, t)^{-1}
$$

The concept of associated volume is a time and depth dependent quantity and can be useful to predict an estimated value of the size of the ejected particles in a mechanism of fast expansion and fragmentation. The ratio of cavity volume over polymer volume (Equation 26) expresses the expansion as a function of depth $z$ and time $t$. 


$$
E_{x p}(z, t)=\frac{v_{c}(z, t)}{v_{a s s}(z, t)}=v_{c}(z, t) \times n_{c}(z, t)
$$

During expansion the cavitating surface develops a uniform network of critical cavities with increasing radius and density. The intercavity space made of polymer is also an expanding network consisting of walls or fibers whose thickness dimension decreases with time and eventually falls to zero due to the stretching phenomena induced by tension. Two particular points of interest can be highlighted, first the interconnection of the cavities and then the spallation. Assuming a cubic network of cavities, at the interconnection point $\left(z_{1}, t_{1}\right)$ the cavity radius meets the relationship expressed by Equation (27) which means that the cavities get in contact to each other. Similarly the expansion ratio has a precise value given in Equation (28).

$$
\begin{gathered}
R\left(z_{1}, t_{1}\right)=\frac{1}{2} n_{c}\left(z_{1}, t_{1}\right)^{-1 / 3} \\
E_{x p}\left(z_{1}, t_{1}\right)=\frac{4 \pi}{3} 0.5^{3}=0.524
\end{gathered}
$$

Most probably in the foam development the point 1 (interconnection) can be exceeded for some particular layer, but at time much larger than the time window of this work. If the conditions are more energetic, then the spallation (point 2) occurs, that is to say expulsion of particles of polymer with small dimension related to $v_{a s s}(z, t)$. Spallation is not the main focus of this study but it is known that in this laser energy regime expulsion of matter by ablation is not negligible. It is reported that spallation is most likely obtained when the expansion factor value is 1 for aluminum (Kuksin \& Yanilin, 2007). However we found that this value is too low and we use a larger value for the reason that the polymer molecules have superior viscoelasticity and can withstand a much larger tension than a liquid metal. This is equivalent to Equation (29)

$$
E_{x p}\left(z_{2}, t_{2}\right) \sim 3
$$

At this point of the research on the laser-induced foaming, we are unable to quantify the respective amounts of ablated polymer and foamed polymer for which we assume that $E_{x p}(z, t)$ is ranging between $0-3$. The values in Table 2 are not larger than 0.12 anyway, meaning that we are exploring only the inception of the cavitation phenomenon and not strictly speaking the bubbling.

\section{Gelatin Film Melting and Cavity Radius Increase}

As indicated in Equation (20) viscosity $\eta(z, t)$ is an important parameter to consider. For polymer at temperature below $T_{\mathrm{g}}$ the solid state is characterized by a viscosity $\eta>10^{12}$ Pa.s a value which makes the frequency factor $Z$ equal to zero. The melting of the gelatin surface in the laser heated layer is necessary for the nucleation of the cavitation. To account for the simultaneous $T$ and $P$ effects on the viscosity $\eta(z, t)$ in the melt layer we use a model (30) adapted from the Avramov equation (AE) established for the glass forming liquids (Avramov, 2000, 2005, 2007). However such model for gelatin is not available and therefore has to be constructed.

$$
\eta(z, t)=\eta_{\infty} \exp \left[30\left(\frac{T_{R}}{T(z, t)}\right)^{\delta_{T}}\left(1+\frac{P(z, t)}{P_{R}}\right)^{\delta_{P}}\right]
$$

In previous work concerning poly(vinyl acetate) (PVAc) (Lazare et al., 2010) the following material parameters were used $\delta_{T}=7.11, \delta_{P}=1.62, T_{R}=302 \mathrm{~K}, P_{R}=2710$ barin the Equation (29) since they have been validated in Roland and Casalini (2003) over a range of pressure 0-5000 bar. It should be pointed out that we assume that such model can be applied in the negative pressure domain of interest in this work. The viscosity $\eta(z, t)$ at the reference temperature $T_{R}=T_{a}$ and $P=1 \mathrm{bar}$ is equal to $10^{12} \mathrm{~Pa}$.s which is the normal viscosity of any polymer (or glass forming liquid) at its glass transition temperature, and the viscosity $\eta_{\infty}$ for infinite temperature is 0.1 Pa.s. Concerning the glass transition of gelatin we take into account the influence of the $5-8 \%$ of water in the film by using the Gordon-Taylor Equation (31) which considers the film as a mixture $\left(T_{g m}\right)$ of the polymer $\left(T_{g p}=466 \mathrm{~K}\right)$ at a fraction $X_{p}=0.92$ and water $\left.T_{g w}=136 \mathrm{~K}\right)$ at a fraction $X_{w}=0.08$ (Roussenova et al., 2012).

$$
T_{g m}=\frac{X_{p} T_{g p}+k_{w} X_{w} T_{g w}}{X_{p}+k_{w} X_{w}}
$$

The constant $k_{w}=0.17$ is the Gordon-Taylor parameter (Díaz et al., 2010) and is obtained by fitting the 
experimental state diagram of gelatin. It is a characteristic of the two constituents gelatin and water and can be related to the ratio of their free volumes, or the ratio of their changes in specific heat across the glass transition (Sobral \& Habitante, 2001). The glass transition temperature of the gelatin films used in the model is $350 \mathrm{~K}$. Above this value the liquid has a characteristic low viscosity at normal pressure of 1 bar. Strictly speaking we do not have access to the gelatin parameters of the Avramov equation, however we chose them in order to describe at best the melting of the films and the pressure/tension dependence. The model of viscosity for gelatin, obtained by similarity with the poly (vinyl acetate) (Lazare et al., 2010), describes the melting range of temperature as displayed in Figure 4. A pressure increase shifts the curve towards larger temperatures whereas a tension shifts it towards smaller temperatures. It means that the high pressure subwave keeps gelatin in the solid state and conversely upon rarefaction the drop down to negative pressure induces melting and the cavities growing up. This is precisely what happens during the transit of the pressure wave over the melt depth (Figure 2).

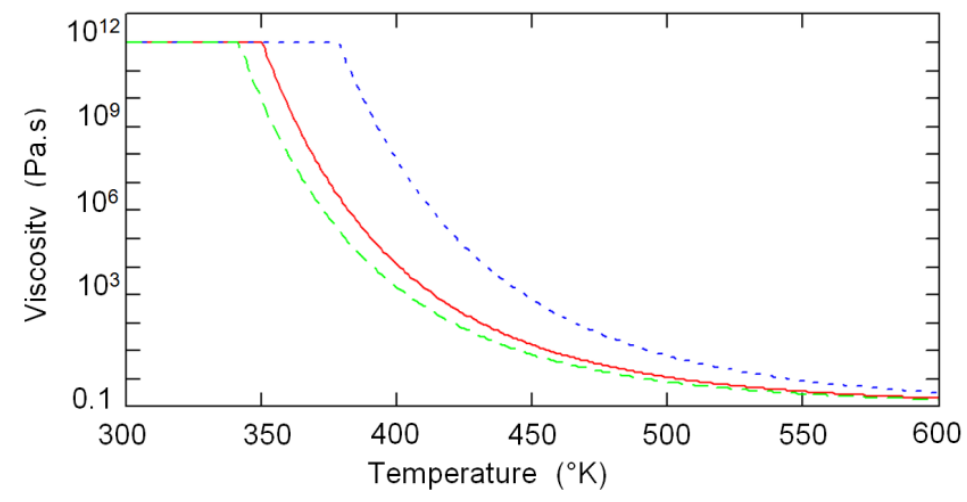

Figure 4. Viscosity of gelatin $\eta$ modeled with the AvramovEquation (29), having high pressure dependence $\delta_{P}=1.5$, for three values of pressure -1000 (dashed green line), +1 (red solid line), +4000 bar (dotted blue line)

For gelatin we use the exponent $\delta_{T}=7.11$, as for PVAc, and the choice of $T_{R}=T_{a}=350 \mathrm{~K}$ is dictated by the glass transition temperature of gelatin for normal pressure. More problematic is the choice of the parameters of the pressure dependence $\delta_{P}$ and $P_{R}$. A strong pressure dependence requires a large $\delta_{P}$ and a low $P_{R}$. However since we are dealing with tensions (negative pressures) of the order of -6000 bar, the ratio $P / P_{R}$ in AE must be larger than -1 , imposing $P_{R}$ of being larger than 6000 bar. Therefore $P_{R}$ is set at a value of 9000 bar. The intensity of the pressure dependence is adjusted in the present model with the parameter $\delta_{P}$ set in the range 0.1 to 1.5 (Figure 4), in order to simulate both low and high pressure dependences. Then by calculating $\eta(z, t)$ as a function $z$ at $t=60 \mathrm{~ns}$, time larger than the end of pulse, for the three experimental fluences, we can theoretically evaluate the thicknesses of the laser-induced melt layer. Figure 5 displays such plots at maximum theoretical temperature. Since we discuss the fluid regime $\left(\eta<10^{12}\right.$ Pa.s) rather than the solid viscosity, we set arbitrarily the solid viscosity at a constant value of $10^{12} \mathrm{~Pa} . \mathrm{s}$ in all this work. As a consequence, the predicted melt depths indicate the cavitation phenomenon is confined in the melt and does not produce in the solid part. As a first remark the higher the fluence the larger melt depth and also the larger tension is developed and the more easily the cavities are produced. On the contrary at the lowest fluence the model predicts almost no melting of the gelatin surface and therefore no cavitation, at least by the present mechanism. We checked for the intensity of the pressure effect on the melt depth and concluded that the influence is small and rather negligible, however the pressure effect on the viscosity of the liquid layer is significant and dependent on the transit of the tension wave. In Figure 6 the viscosity is calculated for the two values of $\delta_{P}=0.1$ and 1.5 as a function of time. For large pressure effect $\delta_{P}=1.5$, it shows that the tension wave induces a drop of viscosity down to values ( $\eta$ is nearing 0.1 Pa.s) indicating good fluidity of the medium, favorable to fast cavitation. 


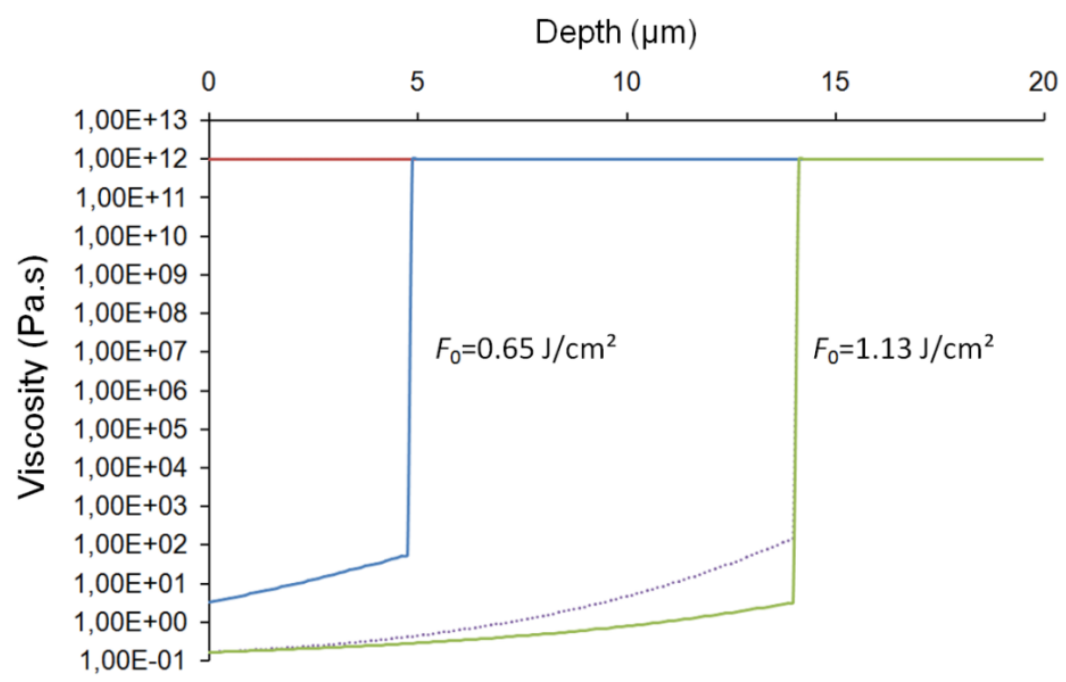

Figure 5. Viscosity $\eta(z, t)$ as a function of depth in the surface of the gelatin film calculated at time $t=60 \mathrm{~ns}$ with Equation 26 for the 3 experimental fluences. The regions of low viscosity at the surface define the melt depths which are $0.0 \mu \mathrm{m}$ for $F_{0}=0.470 \mathrm{~J} / \mathrm{cm}^{2}$ (red line), $4.87 \mu \mathrm{m}$ for $F_{0}=0.650 \mathrm{~J} / \mathrm{cm}^{2}$ (blue line) and $14.1 \mu \mathrm{m}$ for $F_{0}=1.130 \mathrm{~J} / \mathrm{cm}^{2}$ (green line). All solid lines are for high pressure effect $\left(\delta_{P}=1.5\right)$ and the dotted line is for $F_{0}=1.130 \mathrm{~J} / \mathrm{cm}^{2}$ and a low pressure effect $\left(\delta_{P}=0.1\right)$

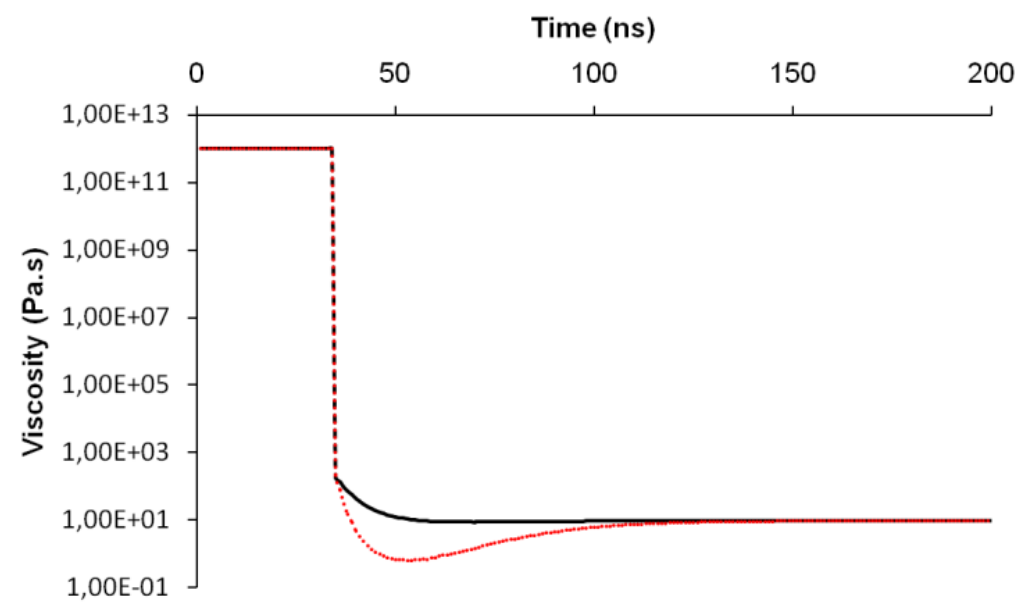

Figure 6. Viscosity $\eta(11 \mu \mathrm{m}, t)$ at depth $11 \mu \mathrm{m}$ as a function of time for a fluence of $F_{0}=1.13 \mathrm{~J} / \mathrm{cm}^{2}$, showing the influence of the tension subwave. The black solid curve is for a low pressure effect $\delta_{P}=0.1$ and the red dotted line is for a stronger pressure effect $\delta_{P}=1.5$ which induces a lower viscosity upon transit of tension

The pressure dependence of viscosity is equally important for the frequency factor $Z(z, t)(20)$ and for the viscous growth of the cavity radius $R(z, t)(32)$. Since both $\Delta P(z, t)(17)$ and $\eta(z, t)(30)$ vary strongly with time, the cavity radius increase is obtained by numerical integration of Equation (23) in our model as with (32):

$$
R(z, t)=R_{0} \int_{0}^{t} \exp \left(\frac{\Delta P\left(z, t^{\prime}\right)}{4 \eta\left(z, t^{\prime}\right)} t^{\prime}\right) d t^{\prime}
$$

The model shows that the cavity growth has some dependence on the pressure effect. In Figure 7 the results for the fluence of $1.13 \mathrm{~J} / \mathrm{cm}^{2}$ indicate that the largest growth occurs at $4 \mu \mathrm{m}$ and reaches a maximum value of $95 \mathrm{~nm}$ at time $150 \mathrm{~ns}$, when the pressure parameter is $\delta_{P}=1.5$. With a small pressure effect $\delta_{P}=0.1$ only, at a depth of $4 \mu \mathrm{m}$ the corresponding cavity radius is reduced to $54.7 \mathrm{~nm}$. The main reason is that viscosity remains higher due to the persistent larger viscosity. In the following only $\delta_{P}=1.5$ is considered. 


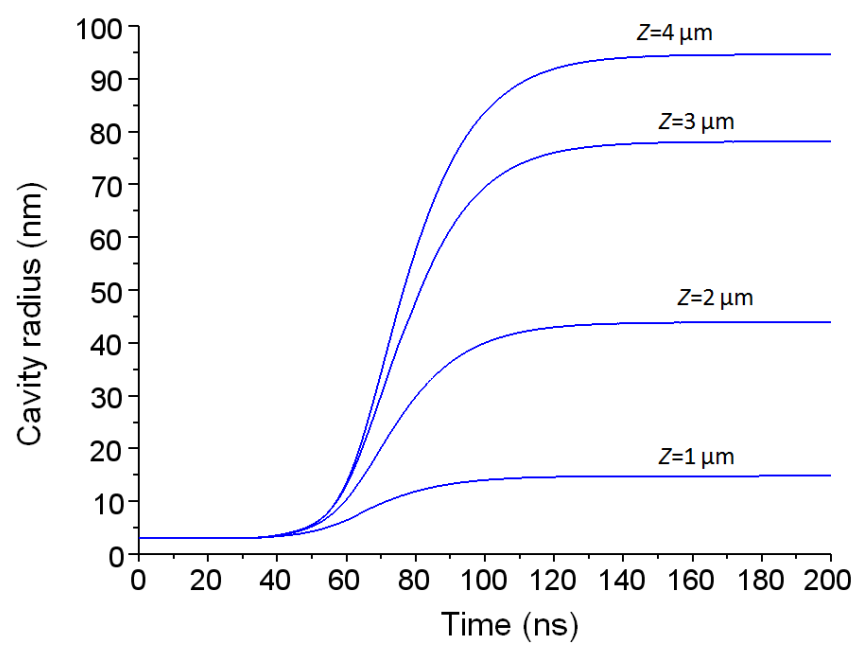

Figure 7. Cavity radius increase as a function of time and depth in the melt depth, $F_{0}=1.13 \mathrm{~J} / \mathrm{cm}^{2}, c_{\mathrm{s}}=1.22$ $\mu \mathrm{m} / \mathrm{ns}$ and $\delta_{P}=1.5$ (high pressure effect on viscosity)

\section{Attenuation Rise Curves}

The model considers the growth of the population of critical cavities during the transit of the pressure wave and more precisely right after the rarefaction wave. Despite the strong non-equilibrium condition due to the pressure wave, this population growth is necessarily limited by the available energy and by the fluctuations of temperature and pressure. It means that the population tends to the maximum density number $n_{0}$ with a rate law expressed by Equation (33) at any z and t. The Rayleigh-Plesset Equation (21) predicts a concomitant radius increase (32) for all cavities assumed by the model. However this growth applies to the critical cavities density only (Equation 33).

$$
n_{c}(z, t)=\int_{0}^{t} J\left(z, t^{\prime}\right) d t^{\prime}
$$

As stated below, the radius increase is limited to the $0-100 \mathrm{~nm}$ range. This allows the use of approximated models, either Rayleigh or Mie, for the calculation of the attenuation coefficient of a single cavity (Cox et al., 2002), since they agree for $R<100 \mathrm{~nm}$ and diverge only for $R>100 \mathrm{~nm}$. However, the Rayleigh one has been preferred in this work, since the Mie model, although having a broader radius range of validity, requires more extensive computation. Each cavity with radius $R(z, t)$, given by Equation (32), attenuates the probe $\mathrm{HeNe}$ laser beam with a cross-sections $(z, t)$ provided by the Rayleigh model (Equation 34 ) where $\lambda_{0}=632.8 \mathrm{~nm}, n_{m}=1.65$ is the refraction index of gelatin, $m=n_{s} / n_{m}$ is the ratio of refractive index of the inner of the spherical cavities $\left(n_{\mathrm{s}}=\right.$ 1) over the refractive index of gelatin $n_{\mathrm{m}}$.

$$
s(z, t)=\frac{8 \pi}{3}\left(\frac{2 \pi n_{m}}{\lambda_{0}}\right)^{4} R(z, t)^{6}\left(\frac{m^{2}-1}{m^{2}+2}\right)^{2}
$$

For the calculation of the total attenuation we consider a single scattering and isolated cavities without light diffusion (Yodh \& Chance, 1995). This approximation is true for small radius and small densities and therefore for small attenuations and less efficient when the radius increases with expansion or when the density of scattering cavities becomes larger. A more complex model capable extending the applicability range is out of the scope of this work. The total attenuation $\operatorname{Att}(t)$ is given by Equation (35) and is confronted with the experimental curves of Figure 1 .

$$
\operatorname{Att}(t)=\frac{I_{0}-I(t)}{I_{0}}=1-10^{-\int_{0}^{\infty} \frac{n_{\mathcal{C}}(z, t) S(z, t)}{\ln (10)} d z}
$$

The main example of predicted attenuation curve is displayed in Figure 8 for the highest value of the parameter $\delta_{P}=1.5$ illustrating the strong influence of pressure and tension on the viscosity. It is seen that the present model of attenuation rise curve closely approaches the experimental one on left only in the case of the highest 
fluence $1.13 \mathrm{~J} / \mathrm{cm}^{2}$. On the opposite with the two other experimental curves of Figure 1 the model does not predict any rise at fluences of $0.650 \mathrm{~J} / \mathrm{cm}^{2}$ and $0.470 \mathrm{~J} / \mathrm{cm}^{2}$. This is due to the absence of melting and the persistent high value of viscosity when the laser fluence is below a threshold $\sim 1.1 \mathrm{~J} / \mathrm{cm}^{2}$, as seen in Figure 4 . However in reality for these lower fluences some opacity rise is actually measured but occurs in much longer times of $0.9 \mu \mathrm{s}$ and $3.5 \mu \mathrm{s}$ respectively. It means that some sort of transition must take place even when the polymer is still in the solid phase involving a different mechanism which we discuss further below. The good approach of Figure 8 was possible by adjusting the effective values of initial cavity radius and concentration at $R_{0}=3 \mathrm{~nm}, n_{0}=5 \times 10^{13} \mathrm{~cm}^{-3}$ which, as we think, is a unique solution for the set of all other parameters used in the model. The adjustment is also in strict compliance with the energy principle presented in the next section. One major concern (see discussion) is the significance of these initial parameters with respect to the approximations and the various system unkowns. These initial values of radius and concentration of cavity precursors $\left(R_{0}, n_{0}\right)$ are far (respectively larger $\times 10$ and smaller $\left.\times 10^{-7}\right)$ from that the free volume holes $\left(R_{f v h}, n_{f v h}\right)$ of gelatin which however can be regarded for comparison. In fact the laser induced-situation is quite different from the room temperature polymer for reasons that are connected to the presence of the ablation gas, the pressure wave and the temperature elevation. The model shows that these effective values $\left(R_{0}, n_{0}\right)$ call for a new mechanistic interpretation.
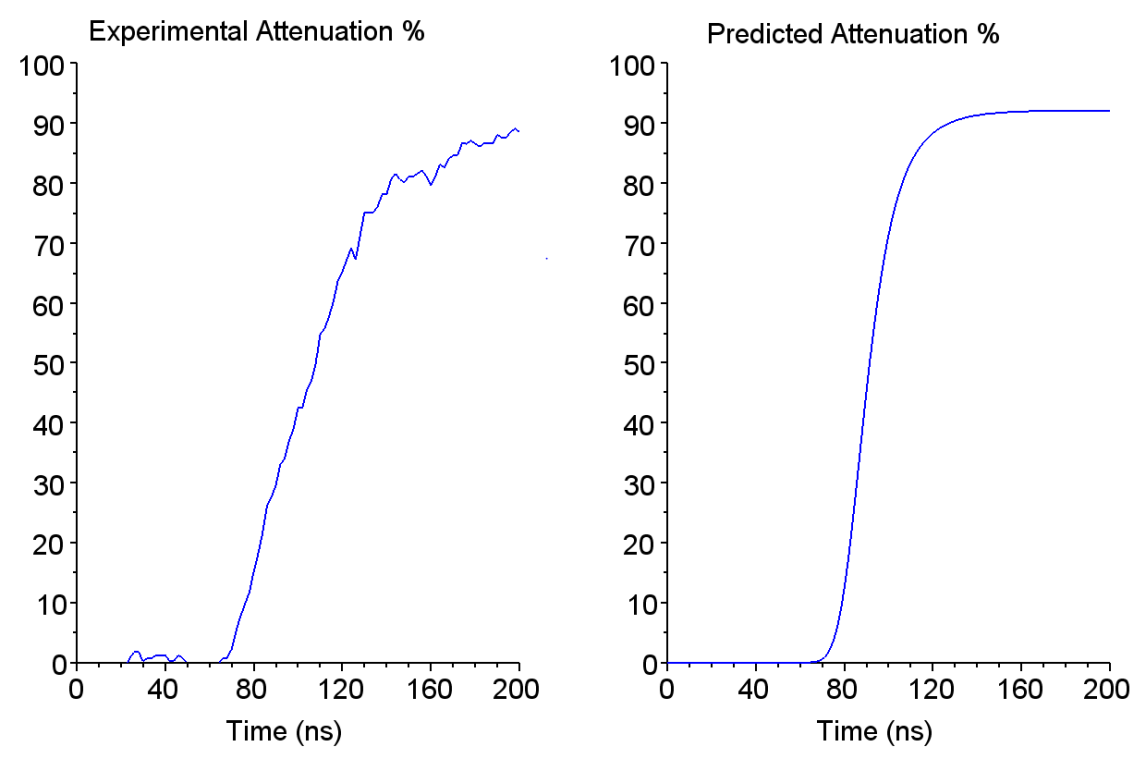

Figure 8. Experimental attenuation rise curve (left) obtained for a fluence of $1.13 \mathrm{~J} / \mathrm{cm}^{2}$ and the predicted attenuation curves (right) calculated at the same fluence with $c_{\mathrm{s}}=1.22 \mu \mathrm{m} / \mathrm{ns}$ and $\delta_{P}=1.5$ and the set effective values of the initial radius and density: $R_{0}=3 \mathrm{~nm}, n_{0}=5 \times 10^{13} \mathrm{~cm}^{-3}$

\section{Limitation Imposed to the Cavitation Energy}

Due to the principle of energy conservation, the computed energy of cavitation $E_{c}(z, t)$ must be kept necessarily smaller than the local input energy $E_{i n}(z, t)$ at any depth value, as expressed in Equation (36).

$$
E_{c}(z, t)<E_{i n}(z, t)=A \alpha F_{0} e^{-\alpha z}
$$




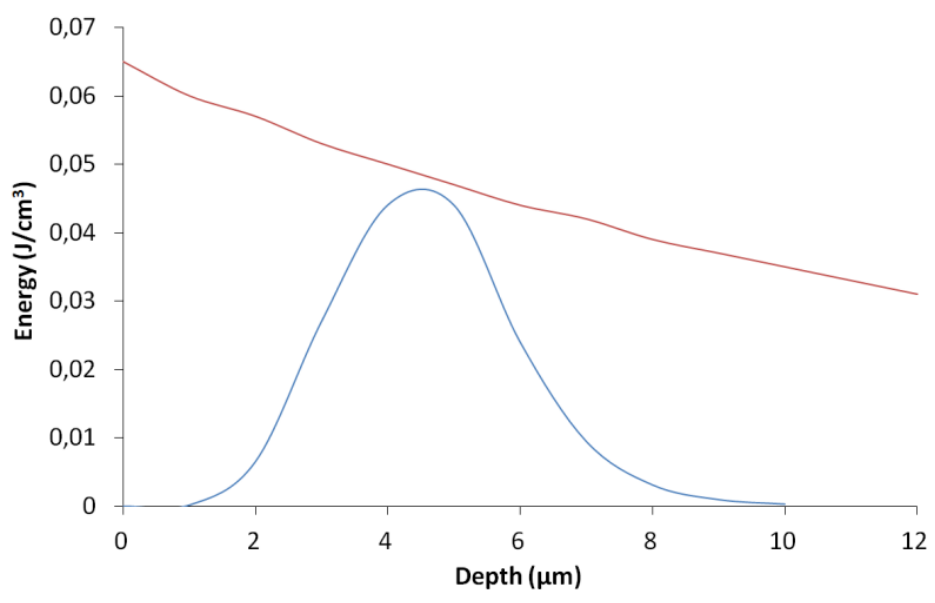

Figure 9. Computed cavitation energy (in blue, Equation 37) compared to the laser input energy (in red, Equation 36) at $F_{0}=1.13 \mathrm{~J} / \mathrm{cm}^{2}$

This is an important guiding for the numerical simulation of the cavitation which can rule out a full range of solutions that consider the enlargement of the too dense network of free volume holes as presented earlier. A more efficient solution appears when a dilute network of bubbles of nanometer initial radius is considered. It is more efficient approach, for meeting the energy limitation and the maximum attenuation $\approx 90 \%$, to envisage a set of larger bubbles with radius $\approx 100 \mathrm{~nm}$ in smaller density rather than tiny cavities in extremely dense number which would require too much energy to create them. The physical reasons behind this spontaneous rise of large bubbles is that cavity coalescence is a very efficient process, preferred in this case, because the ablation gas diffusion is facilitated by the tension state of the polymer. The required energy of cavitation can be estimated by considering the total surface energy since the phenomenon principally produces surface, as displayed in Figure 9. It suffices to consider the time $t=\infty$ ( $\infty$ means $t=150 \mathrm{~ns})$ after the tension wave as in Equation (37).

$$
E_{c}(z, \infty)=n_{c}(z, \infty) \times 4 \pi \times R(z, \infty)^{2} \times \sigma(z, \infty)
$$

\section{Discussion}

For the first time the transient attenuation induced by a short laser pulse is reported along with a cavitation model based on a combined interaction of the viscosity drop (melting), the tension wave and the ablation gases. The measurement recorded for the highest laser fluence $1.13 \mathrm{~J} / \mathrm{cm}^{2}$ is well supported by the above model showing the important role of the "extensive" melting of the surface over a depth of $14.1 \mu \mathrm{m}$ (Figure 5). This liquid layer is enough heated and tensilized to offer the low viscosity which allows the fast cavitation. For lower laser energy the model indicates that the thickness of the molten layer is reduced to $4.87 \mu \mathrm{m}$ for $F_{0}=0.650 \mathrm{~J} / \mathrm{cm}^{2}$ and 0 for $F_{0}=0.470 \mathrm{~J} / \mathrm{cm}^{2}$ and correspondingly no attenuation rise is obtained. In these cases where melting is reduced or absent, it is reasonable to predict no or very little cavitation because the viscosity remains at high values characteristic of either a solid state $F_{0}=0.470 \mathrm{~J} / \mathrm{cm}^{2}$ or a too viscous liquid $F_{0}=0.650 \mathrm{~J} / \mathrm{cm}^{2}$. This means that the cavitation predicted by the model requires the melting of a thick enough layer and the conjonction of a temperature large enough and a strong enough tension wave for the low enough viscosity, conditions that are met only at the highest fluence of $1.13 \mathrm{~J} / \mathrm{cm}^{2}$. The theoretical threshold for such mechanism is $1 \mathrm{~J} / \mathrm{cm}^{2}$ (Table 1) as given by the model. Below this threshold the slower attenuation rise is probably due to a solid phase transition usually characteristic of polymers and named craze phenomenon (Kausch, 1983; James et al., 2012). When solid polymers are subjected to a stress larger than the critical craze value, a typical sort of cavitation may occur usually before large cracks formation and failure. Craze appears in polymers perpendicularly to the main stress direction and is due to the formation of disk-like porous domains whose walls are linked by numerous parallel nano-fibriles spaced by a few nanometers. Craze domains are still mechanically strong but less than the starting polymer and are therefore precursors for cracking and failures. In Table 1 the present model helps clarifying the threshold of fluence $\left(1 \mathrm{~J} / \mathrm{cm}^{2}\right)$ above which the mechanism which we call liquid cavitation takes place. Crazing is expected below this threshold and would be responsible for the slower attenuation rise time. We note that this case is also fluence dependent since crazing is also activated by temperature. Crazing has been extensively studied for many polymers since it is affecting the mechanical resistance of solid polymers whose good understanding is highly relevant to the applications. To the best of our knowledge gelatin films are not fully 
studied yet on the side of mechanical properties and fatigue (Dai \& Liu, 2006). In the laser-induced stress case three factors can contribute to the crazing phenomenon, the pressure wave and mainly the tension tail (i), which also exists below the melting threshold with lower intensity, the ablation gases (ii) which tend to increase the volume and the temperature elevation (iii) which is also expected to activate the process. Crazing, activated by tension and temperature, offers the volume necessary to gas expansion is a likely explanation for the optical attenuation increase measured in the experiment. Even at the low fluence of $0.470 \mathrm{~J} / \mathrm{cm}^{2}$ the optical attenuation is very sensitive to it and the $100 \%$ is reached in $3.5 \mu$ s (Figure 1 and Table 1). For information the amplitude of the tension tail responsible for crazing at $F_{0}=0.470 \mathrm{~J} / \mathrm{cm}^{2}$ reaches a theoretical minimum value of the order of -1750 bar at a depth of 20-30 $\mu \mathrm{m}$ whereas temperature remains below $T_{\mathrm{g}}$. This gives a good estimation of the conditions of crazing but which must be regarded with caution owing to the approximations of the model, the constant Grüneisen coefficient, the neglect of pressure wave attenuation along the propagation direction.

Table 1. Summary of the collected data on the KrF laser-induced dense cavitation in the surface of gelatin

\begin{tabular}{cccccc}
\hline Fluence & $1.13 \mathrm{~J} / \mathrm{cm}^{2}$ & $1.00 \mathrm{~J} / \mathrm{cm}^{2}$ & $0.650 \mathrm{~J} / \mathrm{cm}^{2}$ & $0.500 / \mathrm{cm}^{2}$ & $0.470 \mathrm{~J} / \mathrm{cm}^{2}$ \\
\hline $\begin{array}{c}\text { Model transient melt } \\
\text { layer }\end{array}$ & $14.1 \mu \mathrm{m}$ & $12.0 \mu \mathrm{m}$ & $4.87 \mu \mathrm{m}$ & $\begin{array}{c}\text { Threshold of } \\
\text { surface melting }\end{array}$ & $0 \mu \mathrm{m}$ \\
$\begin{array}{c}\text { Predicted attenuation rise } \\
\begin{array}{c}\text { Observed attenuation } \\
\text { rise }\end{array}\end{array}$ & $\begin{array}{c}110 \mathrm{~ns} \\
(100 \%)\end{array}$ & - & $900 \mathrm{~ns}(100 \%)$ & $\begin{array}{c}\text { Threshold of } \\
\text { foaming }\end{array}$ & $\begin{array}{c}3500 \mathrm{~ns} \\
(100 \%)\end{array}$ \\
$\begin{array}{c}\text { Mechanism } \\
\text { Liquid } \\
\text { cavitation }\end{array}$ & Threshold & Solid crazing & Solid crazing & Solid crazing \\
\hline
\end{tabular}

Table 2 presents detailed results from the model with the initial parameters $\left(R_{0}=3 \mathrm{~nm}, n_{0}=5 \times 10^{-13} \mathrm{~cm}^{-3}\right)$ adjusted in order to provide a good fit of the experimental attenuation curve of figure 8 and a simultaneous cavitation respecting the energy limitation of Figure 9. In the Table 2 the symbol $\infty$ means a time $t \approx 150 \mathrm{~ns}$ which corresponds to the end of the tension wave, as understood by the model. The events occuring at later time are not considered by the model. In particular at much later time the ablation gas may somewhat influence the dynamic of cavity growth, however this knowledge is not yet available. It is observed that the maximum of cavitation is obtained for the layer at depth of $4 \mu \mathrm{m}$ corresponding to the depth with the best combination of low viscosity and strong tension, necessary for a fast cavitation. The near surface layers at depth 1-3 $\mu \mathrm{m}$ are less affected by cavitation mainly because they are exposed to a less intense tension wave, and although they have absorbed more laser energy and they are more fluid (less viscous). Also it is interesting to note that the surface layers are prone to ablation or particle expulsion since they are pushed by the underlying layers, but these events not predicted by the model can be occurring at later time.

Table 2. Computed values of cavity data as a function of depth for the following set of initial values: $\mathrm{F}_{0}=1.13$ $\mathrm{J} / \mathrm{cm}^{2}, c_{s}=1.22 \mu \mathrm{m} / \mathrm{ns}, \delta_{\mathrm{P}}=1.5, R_{0}=3 \mathrm{~nm}, n_{0}=5 \times 10^{-13} \mathrm{~cm}^{-3}$

\begin{tabular}{ccccccccccc}
\hline$z($ depth $\mu \mathrm{m})$ & 1 & 2 & 3 & 4 & 5 & 6 & 7 & 8 & 9 & 10 \\
\hline $\begin{array}{c}n_{c}(z, \infty) \\
\left(\mathrm{cm}^{-3}\right)\end{array}$ & $6.97 \times 10^{12}$ & $2.51 \times 10^{13}$ & $3.05 \times 10^{13}$ & $3.11 \times 10^{13}$ & $2.92 \times 10^{13}$ & $2.55 \times 10^{13}$ & $2.05 \times 10^{13}$ & $1.52 \times 10^{13}$ & $1.01 \times 10^{13}$ & $6.05 \times 10^{13}$ \\
$R(z, \infty)(\mathrm{nm})$ & 14.18 & 43.89 & 78.10 & 94.59 & 93.24 & 68.92 & 44.83 & 27.18 & 16.56 & 10.58 \\
$E_{x p}(z, \infty)$ & 0.001 & 0.009 & 0.0656 & 0.123 & 0.117 & 0.045 & 0.011 & 0.002 & 0.001 & 0.001 \\
\hline
\end{tabular}




\section{Conclusion}

In the experiments dedicated to the study of the mechanism of laser-induced foaming of gelatin film, the fast rise of attenuation before the expansion of the foam is measured for a fluence $F_{0}=1.13 \mathrm{~J} / \mathrm{cm}^{2}$ above the threshold. A model of cavitation including the viscosity and the dynamics and kinetics of the cavity growth is developed with the aim of simulating the attenuation curve (Figure 8). It is shown that in depth melting, viscosity drop and the simultaneous appearance of the tension wave are required to observe a satisfying numerical simulation of the attenuation rise curve. The main initial parameters which had to be adjusted in order to provide a good simulation, are the effective cavity precursor radius $R_{0}=3 \mathrm{~nm}$ and density $n_{0}=5 \times 10^{13} \mathrm{~cm}^{-3}$. This couple of values is the only solution meeting the various criteria of the modeling, experimental dynamics and energy. Comparision with with the free volume hole radius $R_{f v h}=0.227 \mathrm{~nm}$ and density $n_{f v h}=0.5 \times 10^{21} \mathrm{~cm}^{3}$, respectively much smaller and much higher, indicates that ablation gas and cavity coalescence play an important role that should be taken into account in a future modeling. At lower fluence $F_{0}=0.650 \mathrm{~J} / \mathrm{cm}^{2}$, the viscosity model indicates that the melting is limited to a smaller depth which therefore prevents the tension wave to trigger the fast cavitation. Similarly at $F_{0}=0.470 \mathrm{~J} / \mathrm{cm}^{2}$ no melting at all occurs and no tension-induced cavitation is predicted. For these two lower fluences however the slow rise curves measured are attributed to a crazing phenomenon of the gelatin which may well occur at low temperature and in the solid state, mainly due to the formation of the ablation gas.

\section{References}

Akiyama, Y., Shibahara, Y., Takeda, S., Izumi, Y., Honda, Y., Tagawa, S., \& Nishijima, S. (2007). Analysis of swelling process of protein by positron annihilation lifetime spectroscopy and differential scanning calorimetry. J. Pol. Sci. B: Polym. Phys., 45, 2031-2037. http://dx.doi.org/10.1002/pobl.21188

Albagli, D. (1994). Fundamental Mechanisms of Pulsed Laser Ablation of Biological Tissue ( $\mathrm{PhD}$ thesis, Massachussetts Institute of Technology, Cambridge Ma., USA). Retrieved from dspace.mit.edu/bitstream/handle/1721.1/33521/32045390.pdf

Alentiev, A., \&Yampolskii, Y. P. (2002). Meares equation and the role of cohesion energy density in diffusion in polymers. J. Membr. Sci., 206, 291-306. http://dx.doi.org/10.1016/S0376-7388(01)00777-3

Avramov, I. (2000). Pressure dependence of viscosity of glassforming melts. J. Non-Cryst. Sol., 262, 258-263. http://dx.doi.org/10.1016/S0022-3093(99)00712-7

Avramov, I. (2005). Viscosity in disordered media. J. Non-Cryst. Sol., 351, 3163-3173. http://dx.doi.org/10.1016/j.jnoncrysol.2005.08.021

Avramov, I. (2007). Pressure and temperature dependence of viscosity of glassforming and geoscientifically relevant systems. J. Volc. Geotherm. Res., 160, 165-174. http://dx.doi.org/10.1016/j.jvolgeores.2006.09.006

Baidakov, V. G. (2007). Explosive boiling of superheated cryogenic liquids. Berlin, Germany: Wiley-VCH Springer. http://dx.doi.org/10.1002/9783527610662

Castillejo, M., Rebollar, E., Oujja, M., Sanz, M., Selimis, A., Sigletou, M., ... Fotakis, C. (2012). Fabrication of porous biopolymer substrates for cell growth by UV laser: The role of pulse duration. Appl. Surf. Sci., 258, 8919-8927. http://dx.doi.org/10.1016/j.apsusc.2012.05.118

Cox, A. J., DeWeerd, A. J., \& Linden, J. (2002). An experiment to measure Mie and Rayleigh total scattering cross section. Am. J. Phys., 70, 620-625. http://dx.doi.org/10.1119/1.1466815

Dai, C.-A., \& Liu, M.-W. (2006). The effect of crystallinity and aging enthalpy on the mechanical properties of gelatin films. Mat. Sci. Eng. A, 423, 121-127. http://dx.doi.org/10.1016/j.msea.2005.10.082

Dekel, E., Eliezer, S., Henis, Z., Moshe, E., Ludmirsky, A., \& Goldberg, I. B. (1998). Spallation model for the high strain rates range. J. Appl. Phys., 84(9), 4851-4858. http://dx.doi.org/10.1063/1.368727

Díaz, P., López, D., Matiacevich, S., Osorio, F., \& Enrione, J. (2011). State diagram of salmon (Salmosalar) gelatin films. J. Sci. Food. Agric., 91, 2558-2565. http://dx.doi.org/10.1002/jsfa.4451

Dorronsoro, C., Siegel, J., Remon, L., \& Marcos, S. (2008), Suitability of Filofocon A and PMMA for the experimental models in excimer laser ablation refractive surgery. Opt. Express, 16(25), 20955-20967. http://dx.doi.org/10.1364/OE.16.020955

Fisher, B. T., \& Hahn, D. W. (2004). Measurement of small-signal absorption coefficient and absorption cross section of collagen for 193-nm excimer laser light and the role of collagen in tissue ablation. Appl. Opt., 43(29), 5443-5451. http://dx.doi.org/10.1364/AO.43.005443 
Gómez-Guillén, M. C., Giménez, B., López-Caballero, M. E., \& Montero, M. P. (2011). Functional and bioactive properties of collagen and gelatin from alternative sources: A review. Food Hydrocolloids, 25, 1813-1827. http://dx.doi.org/10.1016/j.foodhyd.2011.02.007

James, M. N., Christopher, C. J., Lu, Y., \& Patterson, E. A. (2012). Fatigue crack growth and craze-induced crack tip shielding in polycarbonate. Polymer, 53, 1558-1570. http://dx.doi.org/10.1016/j.polymer.2012.01.032.

Kausch, H. H. (1983). Crazing in Polymers. Adv. Polym. Sci., 52-53, 1-335. Berlin Heidelberg: Springer. http://dx.doi.org/10.1007/BFb0024055

Kuksin, A. Y., Norman, G. E., Pisarev, V. V., Stegalov, V. V., \& Yanilkin, A. V. (2010). Theory and molecular dynamics modeling of spall fracture in liquids. Phys. Rev. B, 82(17), 174101-1-10. http://dx.doi.org/10.1103/PhysRevB.82.174101.

Kuksin, A. Y., \& Yanilin, A. V. (2007). Kinetic model of fracture at high strain rates in the example of high crystalline aluminum. Doklady Phys., 52, 186-190. http://dx.doi.org/10.1134/S1028335807040040

Lazare, S. (2010). Microfoams of biopolymers by laser-induced stretching: mechanisms and applications. In Sciyo (Ed.), Biopolymers, 109-134. http://dx.doi.org/10.5772/10260

Lazare, S., Bonneau, R., Gaspard, S., Oujja, M., De Nalda, R., Castillejo, M., \& Sionkowska, A. (2009). Modeling the dynamics of one laser pulse surface nanofoaming of biopolymers. Appl. Phys. A, 94, 719-729. http://dx.doi.org/10.1007/s00339-008-4950-z

Lazare, S., Elaboudi, I., Castillejo, M., \& Sionkowska, A. (2010). Model properties relevant to laser ablation of moderately absorbing polymers. Appl. Phys. A, 101, 215-224. http://dx.doi.org/10.1007/s00339-010-5754-5

Lazare, S., Tokarev, V., Sionkowska, A., \& Wisniewski, M. (2005). Surface foaming of collagen, chitosan and other biopolymer films by KrFexcimer laser ablation in the photomechanical regime. Appl. Phys. A, 81(3), 465-470. http://dx.doi.org/10.1007/s00339-005-3260-y

Navon, O., Chekhmir, A., \& Lyakhovsky, V. (1998). Bubble growth in highly viscous melts: theory, experiments, and autoexplosivity of dome lavas. Earth and Planetary Sci. Let., 160, 763-776. http://dx.doi.org/10.1016/S0012-821X(98)00126-5.

Oraevsky, A. A., Jacques, S. L., Esenaliev, R. O., \& Tittel, F. K. (1996). Pulsed laser ablation of soft tissues, gels, and aqueous solutions at temperatures below $100{ }^{\circ} \mathrm{C}$. Lasers in Surgery and Medicine, 18, 231-240. http://dx.doi.org/10.1002/(SICI)1096-9101(1996)18:3<231::AID-LSM3>3.0.CO;2-T

Paltauf, G., \& Dyer, P. E. (2003). Photomechanical processes and effects in ablation. Chem. Rev., 103(2), 487-518. http://dx.doi.org/10.1021/cr010436c

Pettit, G. H., \& Ediger, M. N. (1996). Corneal-tissue absorption coefficients for 193- and 213-nm ultraviolet radiation. Appl. Opt., 35(19), 3386-3391. http://dx.doi.org/10.1364/AO.35.003386

Plamann, K., Aptel, F., Arnold, C. L., Courjaud, A., Crotti, C., Deloison, F., ... Salvodelli, M. (2010). Ultrashort pulse laser surgery of the cornea and the sclera. J. Opt., 12(8), 084002. http://dx.doi.org/10.1088/2040-8978/12/8/084002

Plesset, M. S. (1949). The dynamics of cavitation bubbles. J. Appl. Mechan. Trans. ASME, 16, 277-282.

Roland, C. M., \& Casalini, R. (2003). Temperature and Volume Effects on Local Segmental Relaxation in Poly(vinyl acetate). Macromol., 36, 1361-1367. http://dx.doi.org/10.1021/ma025791z

Roussenova, M., Endrione, J., Diaz-Calderon, Taylor, A. J., Ubbink, J., \& Alam, M. A. (2012). A nanostructural investigation of glassy gelatin oligomers: molecular organization and interactions with low molecular weight diluents. New J. Phys., 14, 35016. http://dx.doi.org/10.1088/1367-2630/14/3/035016

Seaman, L., Curran, D. R., \& Shockey, D. A. (1976). Computational models for ductile and brittle fracture. J. Appl. Phys., 47(11), 4814. http://dx.doi.org/10.1063/1.322523

Sobral, P. J. A., \& Habitante, A. M. Q. B. (2001).Phase transitions of pigskin gelatin. Food Hydrocoll., 15, 377-382. http://dx.doi.org/10.1016/S0268-005X(01)00060-1

Trockel, L. S., Srinivasan, R., \& Braren, B. (1983). Excimer laser surgery of the cornea. Amer. J. Ophtalm., 96(6) 710-715.

Vogel, A., \& Venugopalan, V. (2003). Mechanisms of pulsed laser ablation of biological tissues. Chem. Rev., 103(2), 577-644. http://dx.doi.org/10.1021/cr010379n 
Vogel, A., Noack, J., Hüttman, G., \& Paltauf, G. (2005). Mechanisms of femtosecond laser nanosurgery of cells and tissues. Appl. Phys. B: Lasers and Optics, 81(8), 1015-1047. http://dx.doi.org/10.1007/s00340-005-2036-6

Yablon, A. D., Nishioka, N. S., Mikic, B. B., \& Venugopalan, V. (1999). Measurement of tissue absorption coefficients by use of interferometricphotothermal spectroscopy. Appl. Opt., 38(7), 1259-1272. http://dx.doi.org/10.1364/AO.38.001259

Yampolskii, Y. (2010). On estimation of concentration of free volume elements in polymers. Macromol., 43, 10185-10187. http://dx.doi.org/10.1021/ma102332u

Yampolskii, Y. P. (2007). Methods for investigation of the free volume in polymers. Russian Chem. Rev., 76(1), 59-78. http://dx.doi.org/10.1070/RC2007v076n01ABEH003629

Yodh, A, \& Chance, B. (1995). Spectroscopy and Imaging with diffusing light. Phys. Today, 34(3), 34-40. http://dx.doi.org/10.1063/1.881445 